\title{
NEAR INFRARED ELASTIC LIGHT SCATTERING BY A SILICON MICROSPHERE
}

\author{
Ulaş S. Gökay and Ali Serpengüzel \\ Koç University, Microphotonics Research Laboratory, Department of Physics, Rumelifeneri \\ Yolu, Sariyer, Istanbul 34450 Turkey
}

\begin{abstract}
We observed high quality elastic light scattering from a silicon microsphere in the standard telecommunication band. A tunable diode laser was used as the excitation source and a single mode silica optical fiber setup delivered the input laser light to the microsphere. The silicon microsphere was manipulated on the silica optical fiber half coupler (OFHC) to effectively couple the evanescent laser field to the microsphere thus exciting the whispering gallery modes (WGM's). We observed high quality factor WGM's which can lead to novel geometries and applications for silicon microsphere based optoelectronic devices, such as filters, modulators, and detectors.
\end{abstract}

Keywords: elastic light scattering, microsphere, silicon photonics, whispering gallery mode.

\section{INTRODUCTION}

Microcavity resonators employ the light handling properties of the whispering gallery modes (WGM's) of geometrical structures and have been widely researched in recent years ${ }^{1-4}$. Most of the research has focused on the shape of the resonator and uses conventional production techniques to produce the desired cavity structure. As the technology for the device fabrication, equipment handling and light detection matured, researchers started to build increasingly sophisticated systems ${ }^{5-6}$ for creating and manipulating these light resonances for various photonic applications. Not only have the possible resonator geometries such as disks ${ }^{7-8}$, toroids ${ }^{9-10}$ and cylinders ${ }^{11}$ been explored but also various materials such as laser dyes ${ }^{12}$, lithium niobate ${ }^{13}$ and silicon-oninsulator ${ }^{14}$ (SOI) have been experimented with. Each study has improved the research field in the means of smaller geometries and better fabrication techniques, which in turn has lead to increasingly compact and truly sensitive $^{15-16}$ device applications. These device applications range from channel dropping filters ${ }^{17-18}$ to low threshold Raman lasers ${ }^{19}$, electrical and thermo-optical switches ${ }^{20}$ and optical data channel multiplexers ${ }^{21}$. While previous research was a foothold, the current technology is still far from converting electronics into photonics. Manufacturing of such appliances, which will be capable of using light as the incoming data instead of electrons and be able to process the data with full optical or electro-optical circuitry within the structure, is yet to be achieved. Nevertheless prototype devices will be able to perform processing beyond the $\mathrm{GHz}$ frequency regime with their high quality factor resonators and will be consisted of nanometer sized components ${ }^{22}$.

Although current techniques and materials are suitable for today's fabrication needs, distorted geometrical structures and byproducts of materials' fabrication techniques can lead to unintentional Mie and Rayleigh scattering and therefore decreased quality factors ${ }^{13}$ for the WGM's. While spherical microcavities ${ }^{12}$ exhibit some of these drawbacks, they can be fabricated relatively easily. Spherical resonators range from $20 \mu \mathrm{m}$ to $500 \mu \mathrm{m}$ in radius and can be made from various materials. The spherical shape allows light to occupy smaller mode volumes thus enhances the optical quality factor. Together with the spherical geometry, silicon as the cavity material allows building optical devices without adjusting the current semiconductor device fabrication and process technologies.

In this study, we present our results on the observation of high quality factor WGM's in the elastic light scattering spectra of the silicon microspheres by using a near-infrared tunable diode laser used with standard communication optical fibers. Optical resonances with quasi-TM polarization and resulting mode structures were obtained in the $90^{\circ}$ elastic scattering and $0^{\circ}$ transmitted light spectra. The input laser light was spectrally scanned 
and the resonant peaks with a linewidth of $0.9 \mathrm{pm}$ and quality factor of $10^{7}$ were observed. These spectral properties and the coupling scheme used in the experimental setup is beneficial for studying the optical whispering gallery modes of microspheres and can be used for the fabrication of compact channel dropping filters.

\section{EXPERIMENTAL PROCEDURE}

In our experiment, we used standard optical communication fiber optics. The schematic of the whole optical setup can be seen in Fig. 1. A silicon microsphere of radius $\mathrm{a}=500 \mu \mathrm{m}$ was positioned with a three degrees-offreedom translation stage. This unit was then moved over the optical fiber half coupler (OFHC), which contains a stripped single mode optical fiber polished close to the core and placed horizontally through a glass block as seen in the inset of Fig. 1.

The input light was provided by a distributed feedback (DFB) semiconductor diode laser. By using a laser diode controller, the operating wavelength of the laser diode was temperature tuned between $27.31^{\circ} \mathrm{C}$ to $24.29^{\circ} \mathrm{C}$ and the resulting optical power was current adjusted to $500 \mathrm{~mA}$. This temperature range was equivalent to $\Delta \lambda_{\text {range }}=$ $300 \mathrm{pm}$ as calibrated with the Steinhart-Hart equation of the laser diode, while the output optical power of the diode laser was kept constant at $12 \mathrm{~mW}$. The diode laser light was then coupled to the OFHC via an optical fiber polarization controller (P1) to a single mode fiber (SMF). The light in the SMF is assumed to have a Gaussian profile. On the OFHC, a succession of scattering spots provides a guide to where to place the microsphere for evanescent input light coupling. The evanescent coupling of the light from the SMF to the microsphere leads to excitation of the morphology dependent resonances (MDR's) inside the silicon sphere. Elastic scattering resonances with $90^{\circ}$ scattered and $0^{\circ}$ transmitted light were detected with InGaAs photodetectors (PD 1 and PD 2) with a Glan polarizer (P2), while the collection optics (L1, L2 and L3) were used for focusing the optical signal. Transmitted light was measured by an optical wavemeter head (WM 1) and the data acquisition was performed with a digital storage oscilloscope connected to the computer via GPIB interface.

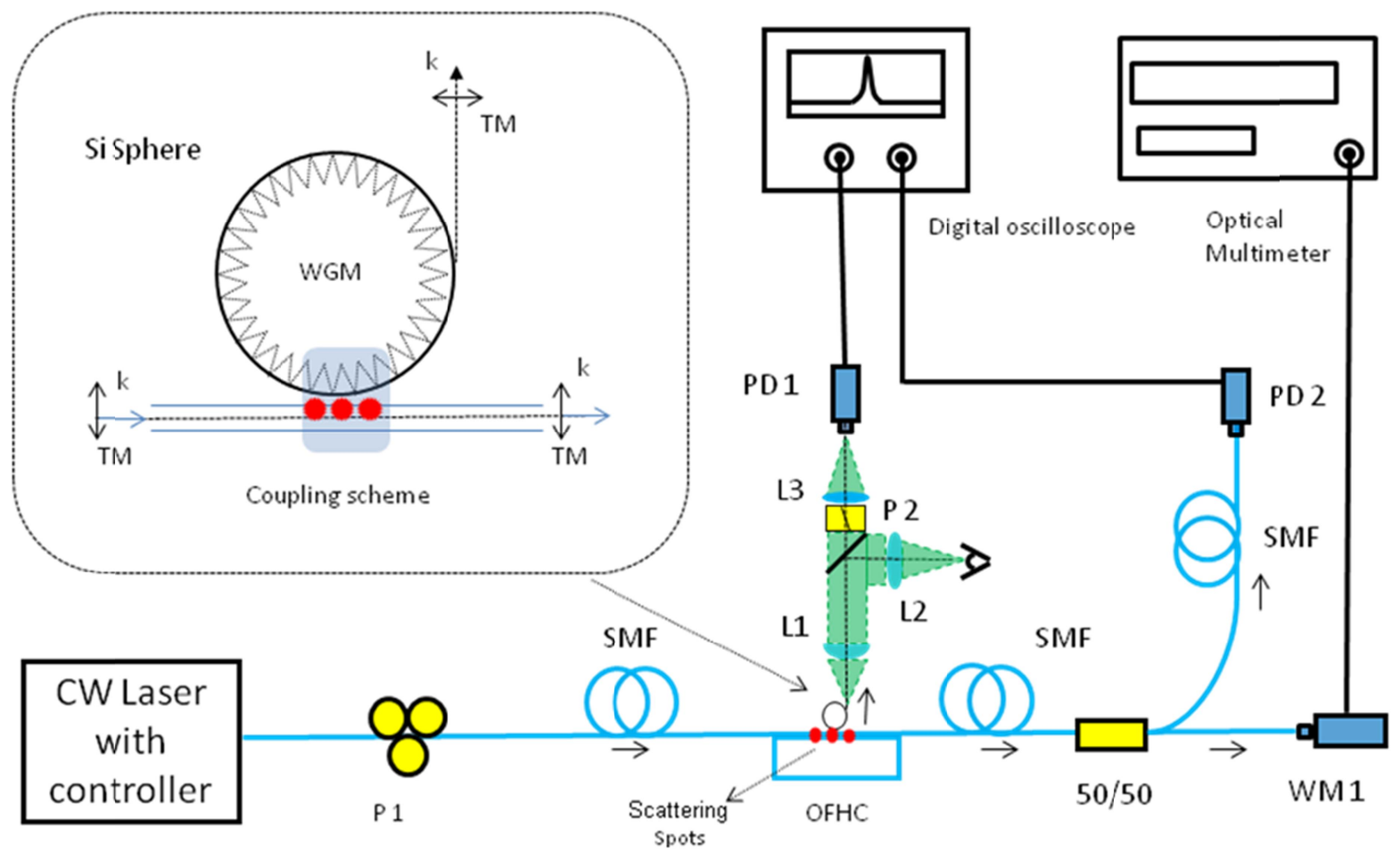

Figure 1. A schematic of the experimental setup. The inset shows polarization arrangement of the silicon sphere over the OFHC, which evanescently couples the light to the sphere with the scattering spots. 


\section{RESULTS AND DISCUSSION}

The elastic light scattering intensities at $90^{\circ}$ and $0^{\circ}$ were collected over a tuning wavelength range of $0.3 \mathrm{~nm}$. The elastic light scattering spectrum shows the expected dips in transmission as the resonance condition is reached and the light is coupled to the microsphere effectively. When one of the scattering spots on the OFHC is in close proximity of the microsphere, the evanescent field is critically coupled to the microsphere resulting in morphology dependent resonances (MDR's). These resonances manifest themselves in mode families separated by the consecutive mode number $(n)$ with the same mode order $(l)$ in the $90^{\circ}$ scattered and $0^{\circ}$ transmitted light spectra (Fig. 2). According to the Lorenz-Mie scattering theory, the mode spacing of MDR's is;

$$
\Delta \lambda=\frac{\lambda^{2} \tan ^{-1} \sqrt{\left(m^{2}-1\right)}}{2 \pi \mathrm{a} \sqrt{\left(m^{2}-1\right)}}
$$

where $\lambda$ is the resonant wavelength, $m$ the relative refractive index and $a$ the radius of the resonator. In our case, $\mathrm{m}=3.5$ and the peak with the best $\mathrm{Q}$ factor is at $\lambda=1427.3143 \mathrm{~nm}$, which yields a mode spacing of $\Delta \lambda=0.2486$ nm. Data in Fig. 2 show 4 mode families in the calculated mode spacing, which can be attributed to the constructive interference of the coupled light in the microresonator ${ }^{18}$. When the input light is in resonance with the microsphere, whispering gallery modes of the microsphere will form a light ring around a great circle of the sphere. Light will also radiate to the outside medium and the fiber coupling via surface of the sphere, thus leading to interference along the resonance spectrum ${ }^{24}$.

Since the MDR's are open systems of light resonances, the energy stored in the resonator cavity will be radiated to the outside medium ${ }^{23}$. This property not only allows the resonance structure to be observed, but also leads to a decreased quality factor in the optical cavity. The quality factor $(Q)$ of an $\operatorname{MDR}$ is;

$$
Q=\frac{\lambda}{\Delta \lambda_{F W H M}}
$$

where $\lambda$ is the resonant wavelength and $\Delta \lambda_{\text {FWHM }}$ the linewidth of the MDR. Figure 2 shows an MDR with a best quality factor of $10^{7}$ at $\lambda=1427.3143 \mathrm{~nm}$ peak with $\Delta \lambda_{\mathrm{FWHM}}=0.9 \mathrm{pm}$. This quality factor mainly depends on the radiative losses of the cavity, which includes absorption, scattering on defects and impurities and the residual surface roughness ${ }^{23}$. Observed $Q$ factor contains contributions from the aforementioned effects, which in turn hinders the storage of the optical energy in the cavity. On the other hand, a silicon microsphere leads to a strong confinement of the light in the inside the cavity medium due to its high refractive index for our wavelength range. As a result, high quality factors for the given structure became attainable. Overall, employment of the silicon as the cavity medium was favorable in terms of high photon confinement, better material handling and the given availability of silicon in our age $\mathrm{e}^{22-23}$. 


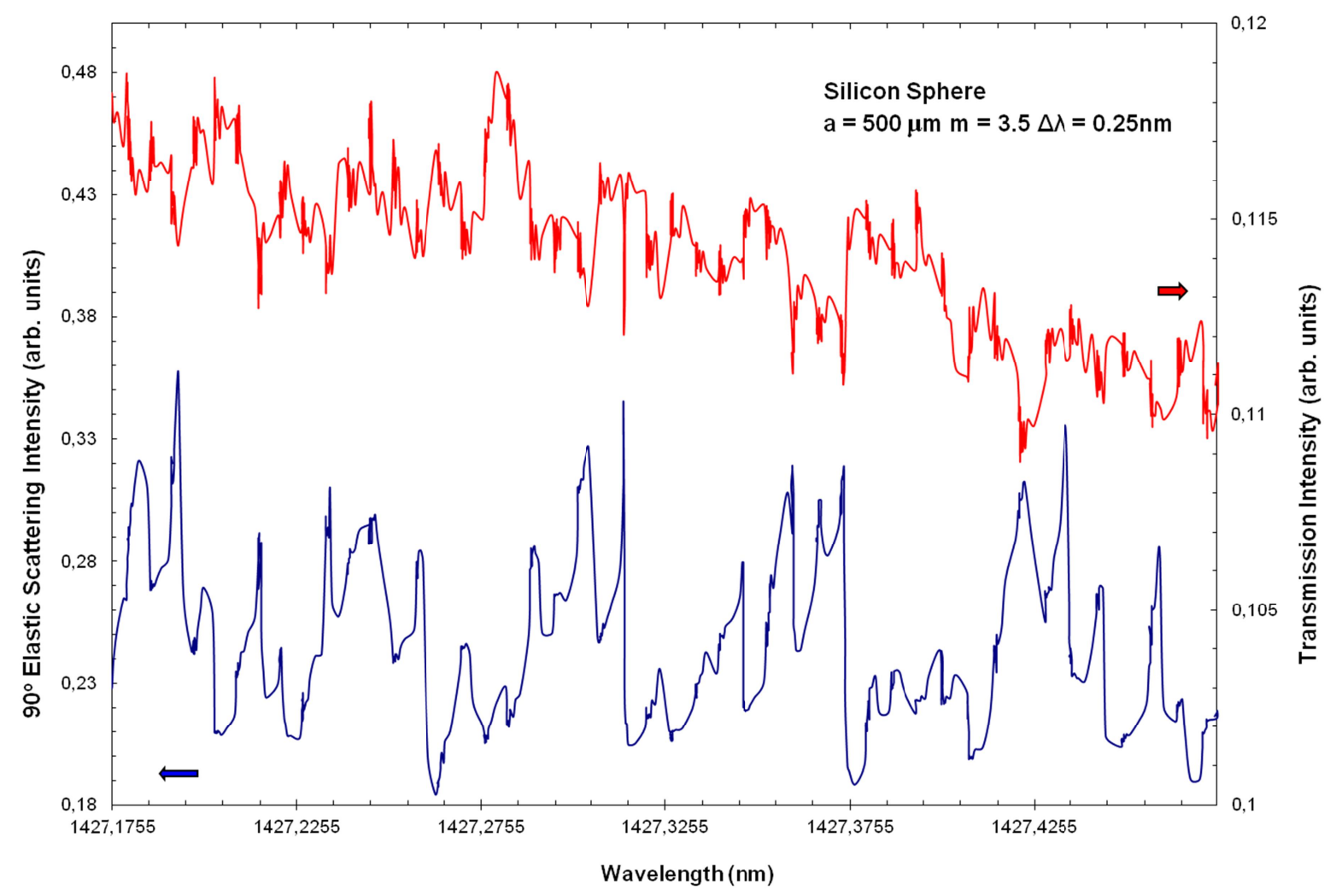

Figure 2. Transmission and $90^{\circ}$ elastic light scattering spectra from a silicon sphere for quasi-TM polarization.

\section{CONCLUSIONS}

In this study, we present high quality factor resonances in the elastic light scattering from a silicon microsphere. The input light was obtained by a near infrared diode laser connected via conventional fiber optics. The coupling of the light to the microsphere was achieved by using an optical fiber half coupler. The polarization dependence and the spectral properties were measured in $90^{\circ}$ elastic scattering and $0^{\circ}$ transmitted output light. High quality factor $\left(10^{7}\right)$ resonances were observed by an optimized coupling scheme. Overall experimental results were analyzed by the Lorenz-Mie scattering theory. The results are promising for compact device applications for channel drop filtering and wavelength division multiplexing.

\section{REFERENCES}

[1] Vahala, K., Herrmann, M., Knünz, S., Batteiger, V., Saathoff, G., Hänsch, T. W., and Udem, T., "A phonon laser," Nature Physics 5, 682-686 (2009).

[2] Wen, Y. H., Kuzucu, O., Fridman, M., Gaeta, A. L., Luo, L. W., and Lipson, M., "All-optical control of an individual resonance in a silicon microresonator," Physical Review Letters, 108(22), 223907 (2012).

[3] Wang, Q. J., Yan, C., Yu, N., Unterhinninghofen, J., Wiersig, J., Pflügl, C., and Capasso, F., "Whisperinggallery mode resonators for highly unidirectional laser action," Proceedings of the National Academy of Sciences, 107(52), 22407-22412 (2010).

[4] Özdemir, S. K., He, L., Zhu, J., Monifi, F., Kim, W., Kenechukwu O., Yılmaz, H., Huang, S. H., Yang, L., "On-chip whispering-gallery-mode microlasers and their applications for nanoparticle sensing," in Proceedings of SPIE 8627, Integrated Optics: Devices, Materials, and Technologies XVII, 86270N (2013). 
[5] Schietinger, S., and Benson, O., "Coupling single NV-centres to high-Q whispering gallery modes of a preselected frequency-matched microresonator," Journal of Physics B: Atomic, Molecular and Optical Physics, 42(11), 114001 (2009).

[6] O'Shea, D., Rettenmaier, A., and Rauschenbeutel, A., "Active frequency stabilization of an ultra-high Q whispering-gallery-mode microresonator," Applied Physics B: Lasers and Optics, 99(4), 623-627 (2010).

[7] Borselli, M., Srinivasan, K., Barclay, P. E., and Painter, O., "Rayleigh scattering, mode coupling, and optical loss in silicon microdisks," Applied Physics Letters, 85(17), 3693-3695 (2004).

[8] McCall, S. L., Levi, A. F. J., Slusher, R. E., Pearton, S. J., and Logan, R. A., "Whispering gallery mode microdisk lasers," Applied Physics Letters, 60 (3), 289-291 (1992).

[9] Armani, D. K., Kippenberg, T. J., Spillane, S. M., and Vahala, K. J., "Ultra-high-Q toroid microcavity on a chip," Nature, 421(6926), 925-928 (2003).

[10] Kippenberg, T. J., Spillane, S. M., and Vahala, K. J., "Kerr-nonlinearity optical parametric oscillation in an ultrahigh-Q toroid microcavity," Physical Review Letters, 93(8), 83904 (2004).

[11] White, I. M., Oveys, H., and Fan, X., "Liquid-core optical ring-resonator sensors," Optics Letters, 31(9), 1319-1321 (2006).

[12] Tzeng, H. M., Wall, K. F., Long, M. B. and Chang, R. K., "Laser emission from individual droplets at wavelengths corresponding to morphology-dependent resonances," Optics Letters, 9(11), 499-501 (1984).

[13] Fürst, J. U., Strekalov, D. V., Elser, D., Aiello, A., Andersen, U. L., Marquardt, C., and Leuchs, G., "Lowthreshold optical parametric oscillations in a whispering gallery mode resonator," Physical Review Letters, 105(26), 263904 (2010).

[14] Baehr-Jones, T., Hochberg, M., Walker, C., and Scherer, A., "High-Q optical resonators in silicon-oninsulator-based slot waveguide," Applied Physics Letters, 86(8), 081101 (2005).

[15] Chembo, Y. K., Baumgartel, L., and Yu, N., "Exploring the frequency stability limits of whispering gallery mode resonators for metrological applications," in Proceedings of SPIE Vol. 8236, Laser Resonators, Microresonators, and Beam Control XIV, 82360Q, (2012).

[16] Dong, P., Liao, S., Liang, H., Qian, W., Wang, X., Shafiiha, R., and Asghari, M., "High-speed and compact silicon modulator based on a racetrack resonator with a 1 V drive voltage," Optics Letters, 35(19), 3246-3248 (2012).

[17] Prabhu, A. M., Tsay, A., Han, Z., and Van, V., "Ultracompact SOI microring add-drop filter with wide bandwidth and wide FSR," Photonics Technology Letters, IEEE, 21(10), 651-653 (2009).

[18] Bilici, T., Işçi, S., Kurt, A., and Serpengüzel, A., "Microsphere-based channel dropping filter with an integrated photodetector," Photonics Technology Letters, IEEE, 16(2), 476-478 (2004).

[19] Galushkin, M. G., Mitin, K. V., and Seryogin, A. M., "Low threshold Raman laser," in Proceedings of the SPIE Vol. 7993, ICONO 2010: International Conference on Coherent and Nonlinear Optics, 799330V, (2010).

[20] Yılmaz, H., Tamer, M. S., Gürlü, O., and Serpengüzel, A., "Tuning optical resonances of a microsphere with liquid crystal," in Proceedings of SPIE Vol. 8069, Integrated Photonics: Materials, Devices, and Applications, 806917, (2011).

[21] Situ, G., and Zhang, J., "Multiple-image encryption by wavelength multiplexing," Optics Letters, 30(11), 1306-1308 (2005). 
[22] Davis, A., Jouppi, N. P., McLaren, M., Muralimanohar, N., Schreiber, R. S., Binkert, N., and Ahn, J. H., [The Role of Photonics in Future Datacenter Networks], in Optical Interconnects for Future Data Center Networks, pp. 67-93, Springer, New York (2005).

[23] Righini, G. C., Dumeige, Y., Féron, P., Ferrari, M., Nunzi Conti, G., Ristic, D., and Soria, S., "Whispering gallery mode microresonators: fundamentals and applications," Rivista del Nuovo Cimento, 34(7), 435-488 (2011).

[24] Schiller, S. and Byer, R. L., "High-resolution spectroscopy of whispering gallery modes in large dielectric spheres," Optics Letters, 16(15), 1138-1140 (1991). 